\title{
ESTUDIO COMPARATIVO DE LOS EFECTOS DE DOS DROGAS ANTIGLAUCOMATOSAS SOBRE EL ESPESOR CORNEAL EN CONEJOS CON AUTOINJERTO DE CÓRNEA
}

\section{COMPARATIVE STUDY OF THE EFFECTS OF TWO ANTI- GLAUCOMATOUS DRUGS ON THE CORNEAL THICKNESS IN RABBITS WITH CORNEAL AUTOGRAFTS}

\author{
CHIARADIA PA ${ }^{1}$, CASIRAGHI JF², FISCHBARG J ${ }^{3}$, LAVENA PI ${ }^{4}$
}

\section{RESUMEN}

Objetivo: Determinar las variaciones del espesor centrocorneal tras la administración de dos drogas antiglaucomatosas: latanoprost $0,005 \%$ o dorzolamida $2 \%$ en conejos con auto injerto de cornea de espesor total.

Métodos: Diez conejos recibieron un autoinjerto de córnea de espesor total bilateralmente. Un animal fue excluido del estudio. En los nueve restantes, a los dos meses de la intervención se instauró tratamiento con un fármaco antiglaucomatoso tópico. El ojo derecho recibió latanoprost al $0,005 \%$, una vez al día, y el ojo izquierdo recibió dorzolamida al $2 \%$ cada 12 horas. Los ojos fueron examinados $0,4,10$, 17 y 27 semanas después del inicio del tratamiento con fármacos mediante biomicroscopia y paquimetría ultrasónica. En cada tiempo examinado, se realizaron 3 medidas del espesor central corneal en cada ojo.

Al final del estudio se analizó la influencia del tiempo y del tratamiento sobre el espesor corneal utili-

\begin{abstract}
Purpose: To determine the central corneal thickness after administration of the anti-glaucomatous medications latanoprost $0.005 \%$ or dorzolamide $2 \%$, as assessed in rabbits which have had total corneal thickness autografts.

Methods: A bilateral total corneal thickness autograft was performed in ten rabbits. One rabbit was excluded from the subsequent study in which the antiglaucomatous medication was started two months post-operatively. Latanoprost $0.005 \%$ was instilled once per day into the right eye, whereas the left eyes were treated with dorzolamide $2 \%$ twice a day. The eyes were examined by biomicroscopy and ultrasound pachymetry immediately prior to commencement, and 4, 10, 17 and 27 weeks after starting the anti-glaucomatous treatment. In each instance three assessments of the central corneal thickness in each eye were made. At the end of the study, the influence of time and treatment on the corneal thickness was analyzed using a generalized
\end{abstract}

\footnotetext{
Recibido: 9/12/05. Aceptado: 6/3/08.

Hospital de Clínicas José de San Martín. Universidad de Buenos Aires. Buenos Aires. Argentina.

1 Doctor en Medicina. Jefe de la Sección de Córnea. Servicio de Oftalmología.

2 Doctor en Medicina. Jefe de la Sección de Glaucoma. Servicio de Oftalmología.

3 Doctor en Medicina. Profesor de la Universidad de Columbia. Columbia University. Medical Center, College of Physicians and Surgeons.

Nueva York, Estados Unidos.

4 Doctor en Medicina. Médica de planta.

Se contó con financiación parcial del NIH Grant EY061178 (JF).
}

Correspondencia:

Pablo Chiaradia

Arenales, 1733 - $\mathrm{PB} \ll \mathrm{A} »$

1061 Buenos Aires

Argentina

E-mail: pchiaradia@fibertel.com.ar 
zando un modelo lineal generalizado de medidas repetidas. Todas las queratoplastias penetrantes fueron efectuadas por el mismo cirujano (CHP).

Resultados: El tratamiento con dorzolamida produjo edema corneal y un aumento significativo del espesor corneal central mientras que el tratamiento con latanoprost no produjo edema corneal ni modificó el espesor corneal.

Conclusión: La dorzolamida, aplicada sobre ojos de conejo con autoinjerto de córnea tiene un efecto negativo sobre el injerto, posiblemente empeorando la función del endotelio corneal mediante la inhibición de la bomba iónica. Este efecto puede evaluarse mediante paquimetría ultrasónica y podría provocar un fallo del injerto.

Palabras claves: Espesor corneal, latanoprost $0,005 \%$, dorzolamida $2 \%$, paquimetría. linear model for repeated measurements. All penetrating keratoplasties were performed by the same surgeon (C.H.P).

Results: Treatment with dorzolamide resulted in corneal edema and a significant increase in central corneal thickness, whereas the treatment with latanoprost resulted in neither corneal edema nor corneal thickness changes.

Conclusions: Dorzolamide, when instilled into the eyes of rabbits with corneal autografts, could have a negative effect on the graft, impairing the endothelial function through inhibition of the ionic pump. This effect could cause graft failure, which may be able to be defined with ultrasound pachimetry (Arch Soc Esp Oftalmol 2008; 83: 257-262).

Key words: Corneal thickness, latanoprost $0.005 \%$, dorzolamide $2 \%$, pachymetry.

\section{INTRODUCCIÓN}

El incremento de la presión intraocular es una complicación frecuente tras la queratoplastia penetrante, siendo en muchas ocasiones de difícil control.

Los parámetros farmacocinéticos y rangos de seguridad de las drogas antiglaucomatosas en corneas normales son bien conocidas resultando ser estas medicaciones seguras y efectivas.

El efecto negativo de los inhibidores de la anhidrasa carbónica (IAC) por vía tópica sobre la bomba endotelial corneal ha sido objeto de estudio, mostrando la seguridad del fármaco sobre córneas sanas, en relación a la transparencia, grosor y celularidad endotelial (1-5).

Los IAC son capaces de reducir la formación de iones bicarbonato de la célula, disminuyendo el transporte asociado de sodio y agua, pudiendo así producir en ojos con función endotelial deficiente un edema corneal.

In vivo se puede evaluar de manera indirecta el funcionamiento del endotelio corneal con la medida del espesor corneal; es entonces la paquimetría ultrasónica un método útil para la observación de pacientes que han sido sometidos a queratoplastia penetrante (6). Existe una relación lineal entre hidratación y espesor corneal, al ser el espesor central de un injerto una medida clínicamente relevante para evaluar un transplante.
Dado que las variaciones del espesor corneal son una medida indirecta de insuficiencia endotelial, en este estudio se ha realizado un análisis del las variaciones del espesor corneal en ojos de conejos tras queratoplastia penetrante y la administración de dos drogas antiglaucomatosas: dorzolamida $2 \%$ y latanoprost $0,005 \%$ y como se caracteriza la evolución de estos efectos.

\section{SUJETOS, MATERIAL Y MÉTODOS}

Se han utilizado diez conejos de entre doce y quince meses, albinos, raza Nueva Zelanda, de un peso entre 1,5 y $1,7 \mathrm{~kg}$, obtenidos del criadero Gilardoni S.A., General Rodríguez, provincia de Buenos Aires, Argentina. Todas las manipulaciones experimentales de los animales se realizaron siguiendo las normas de experimentación animal dictadas por la Administración Nacional de Medicamentos, Alimentos y Tecnología Médica (ANMAT), el servicio Nacional de Sanidad y Calidad Agroalimentaria (SENASA) y la Facultad de Ciencias Exactas y Naturales de la Universidad de Buenos Aires. Los animales fueron anestesiados con la aplicación de clorhidrato de xilocina al $2 \%$, Rompun $^{\circledR}$ (Bayer), $5 \mathrm{mg} / \mathrm{kg}$ por vía subcutánea y Ketamina, Ketalar ${ }^{\circledR}$ (Parque Davis), $30 \mathrm{mg} / \mathrm{kg}$ por vía intramuscular, realizándoseles un autoinjerto de córnea bilateral de espesor penetrante de $7 \mathrm{~mm}$ de 
diámetro. La trepanación se realizó a mano alzada con trépanos de mano (Trephine handle) de la casa Storz instrument company F3096 de 7 mm (St. Louis, Missouri); y los injertos fueron suturados con nylon 10-0, (Alcon Inc., USA).

Tras 2 meses de la intervención se instauró tratamiento con un fármaco antiglaucomatoso tópico en ambos ojos: el ojo derecho recibió latanoprost al 0,0005\%, Xalatan ${ }^{\circledR}$ (Pharmacia, Bélgica), una vez al día y el ojo izquierdo recibió dorzolamida al 2\% Trusopt $^{\circledR}$ (Merck Sharp \& Dohme-Chibert, Clermont - Ferrand, France), cada 12 horas. Los ojos de los animales fueron examinados 0, 4, 10, 17 y 27 semanas después del inicio del tratamiento mediante biomicroscopia y paquimetría ultrasónica (Paquímetro BVI. Model Pocket - BV Internacional Clermont - Ferrand, France). Las medidas del espesor corneal fueron volcadas en una hoja de cálculo del Exel ${ }^{\circledR}$, versión office XP, Microsoft Corp. y analizadas con el programa estadístico SPSS, versión 11.5. (SPSS Inc, Illinois, USA).

Al final del estudio se analizó la influencia del tiempo y del tratamiento sobre el espesor corneal utilizado un modelo lineal generalizado de medidas repetidas.

\section{RESULTADOS}

Un animal fue excluido porque desarrolló una endoftalmitis en el ojo izquierdo a 8 semanas tras la intervención.

En los nueve animales restantes, la evaluación biomicroscópica se realizó en las semanas $0,4,10$, 17 y 27 y mostró edema corneal que se acentuó progresivamente en los ojos tratados con dorzolamida $2 \%$ (figs. 1 y 2 ).

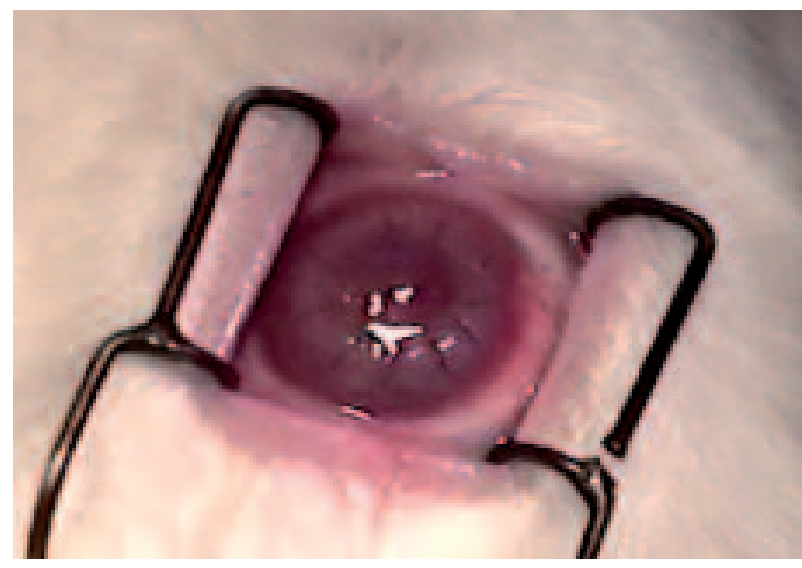

Fig. 1: Ojo de conejo a las 27 semanas de aplicación de latanoprost 0,005\%. 1 vez por día.

Mediante paquimetría ultrasónica se pudo objetivar dicho engrosamiento corneal (tabla I, fig. 3).

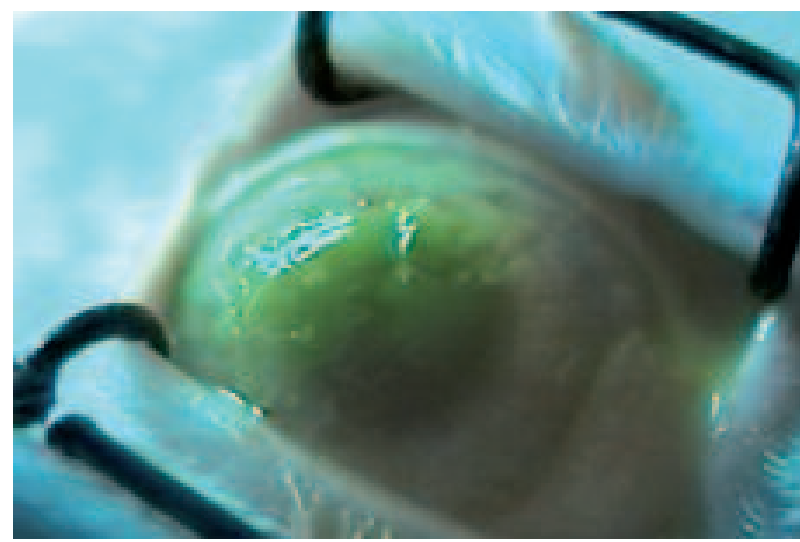

Fig. 2: Los ojos con queratoplastia, a los que se les suministró Dorzolamida al $2 \%$ evolucionaron hacia edema corneal.

Tabla I. Medidas descriptivas y evolución temporal

\begin{tabular}{|c|c|c|c|c|c|}
\hline \multirow[t]{2}{*}{ Ojos } & \multirow[t]{2}{*}{ Tiempo } & \multirow[t]{2}{*}{ Media } & \multirow[t]{2}{*}{ Error típ. } & \multicolumn{2}{|c|}{ Intervalo de confianza al $95 \%$} \\
\hline & & & & Límite inferior & Límite superior \\
\hline \multirow[t]{5}{*}{ Derecho $(n=9)$} & Basal & 564,963 & 46,764 & 457,126 & 672,800 \\
\hline & 4 semanas & 554,481 & 43,836 & 453,395 & 655,568 \\
\hline & 10 semanas & 556,593 & 43,776 & 455,646 & 657,539 \\
\hline & 17 semanas & 555,593 & 40,763 & 461,593 & 649,592 \\
\hline & 27 semanas & 556,815 & 39,538 & 465,640 & 647,989 \\
\hline \multirow[t]{5}{*}{ Izquierdo $(\mathrm{n}=9)$} & Basal & 573,667 & 47,287 & 464,623 & 682,711 \\
\hline & 4 semanas & 636,296 & 36,976 & 551,029 & 721,563 \\
\hline & 10 semanas & 646,481 & 32,346 & 571,890 & 721,073 \\
\hline & 17 semanas & 712,000 & 48,792 & 599,484 & 824,516 \\
\hline & 27 semanas & 714,370 & 45,140 & 610,277 & 818,463 \\
\hline
\end{tabular}




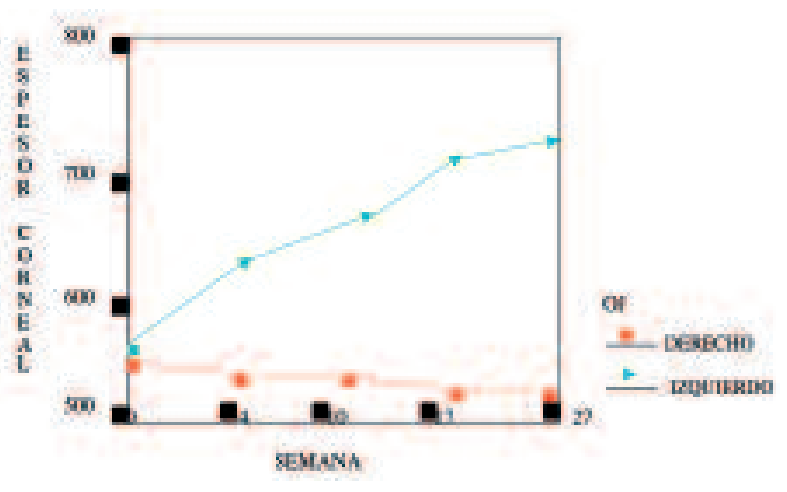

Fig. 3: Evolución temporal de cada ojo. En ordenadas figura el factor espesor corneal y en abscisas el factor tiempo, observándose las diferencias tras la administración de 2 drogas (dorzolamida 2\% y latanoprost $0,005 \%$ ).

Asimismo se pudo observar que no hubo variaciones estadísticamente significativas en el espesor corneal de conejos tratados con latanoprost $0,0005 \%$.

Para el análisis estadístico de la evolución del espesor corneal (tablas II y III), se consideraron la influencia del tiempo y del tratamiento y un tercer efecto de medidas repetidas que permite considerar las tres mediciones en cada instancia. El modelo propuesto y probado incluye el efecto de los factores tiempo y ojos y la interacción entre ambos (fig. 3 y tablas I, II y III).

\section{DISCUSIÓN}

Los inhibidores de la anhidrasa carbónica tópicos alteran la función endotelial a largo plazo como evidencia la literatura, en corneas con queratopatías o cirugías oculares.

Sabemos que la bomba endotelial es efectiva tras la administración de IAC tópicos en corneas sanas

Tabla II. Evaluación de la significación estadística. Contrastes multivariados (c)

\begin{tabular}{llrrrrc}
\hline Efecto & Tests utilizados & Valor & F & Gl de la hipótesis & Gl del error & Significación \\
\hline \multirow{2}{*}{ TIEMPO } & Traza de Pillai & 0,822 & $5,785(\mathrm{~b})$ & 4,000 & 5,000 & $0,041^{*}$ \\
& Lambda de Wilks & 0,178 & $5,785(\mathrm{~b})$ & 4,000 & 5,000 & $0,041^{*}$ \\
& Traza de Hotelling & 4,628 & $5,785(\mathrm{~b})$ & 4,000 & 5,000 & $0,041^{*}$ \\
\multirow{3}{*}{ OJOS } & Raíz mayor de Roy & 4,628 & $5,785(\mathrm{~b})$ & 4,000 & 5,000 & $0,041^{*}$ \\
& Traza de Pillai & 0,606 & $12,285(\mathrm{~b})$ & 1,000 & 8,000 & $0,008^{* *}$ \\
& Lambda de Wilks & 0,394 & $12,285(\mathrm{~b})$ & 1,000 & 8,000 & $0,008^{* *}$ \\
\multirow{2}{*}{ TIEMPO * OJOS } & Traza de Hotelling & 1,536 & $12,285(\mathrm{~b})$ & 1,000 & 8,000 & $0,008^{* *}$ \\
& Raíz mayor de Roy & 1,536 & $12,285(\mathrm{~b})$ & 1,000 & 8,000 & $0,008^{* *}$ \\
& Traza de Pillai & 0,884 & $9,543(\mathrm{~b})$ & 4,000 & 5,000 & $0,015^{*}$ \\
& Lambda de Wilks & 0,116 & $9,543(\mathrm{~b})$ & 4,000 & 5,000 & $0,015^{*}$ \\
& Traza de Hotelling & 7,634 & $9,543(\mathrm{~b})$ & 4,000 & 5,000 & $0,015^{*}$ \\
& Raíz mayor de Roy & 7,634 & $9,543(\mathrm{~b})$ & 4,000 & 5,000 & $0,015^{*}$ \\
\hline \hline
\end{tabular}

* Significativo al 5\%; ** Significativo al $1 \%$.

Tabla III. Pruebas de efectos intra-sujetos

\begin{tabular}{llcrrrc}
\hline Fuente & \multicolumn{1}{c}{ Tests utilizados } & Suma de cuadrados tipo III & gl & Media cuadrática & F & Significación \\
\hline TIEMPO & Esfericidad asumida & 168498,015 & 4 & 42124,504 & 5,147 & $0,003^{* *}$ \\
& Greenhouse-Geisser & 168498,015 & 1,237 & 136176,832 & 5,147 & $0,041^{*}$ \\
& Huynh-Feldt & 168498,015 & 1,351 & 124723,957 & 5,147 & $0,037^{*}$ \\
\multirow{2}{*}{ OJOS } & Límite-inferior & 168498,015 & 1,000 & 168498,015 & 5,147 & 0,053 \\
& Esfericidad asumida & 659885,570 & 1 & 659885,570 & 12,285 & $0,008^{* *}$ \\
& Greenhouse-Geisser & 659885,570 & 1,000 & 659885,570 & 12,285 & $0,008^{* *}$ \\
\multirow{5}{*}{ TIEMPO * OJOS } & Huynh-Feldt & 659885,570 & 1,000 & 659885,570 & 12,285 & $0,008^{* *}$ \\
& Límite-inferior & 659885,570 & 1,000 & 659885,570 & 12,285 & $0,008^{* *}$ \\
& Esfericidad asumida & 205956,652 & 4 & 51489,163 & 8,778 & $0,000^{* *}$ \\
& Greenhouse-Geisser & 205956,652 & 1,404 & 146724,500 & 8,778 & $0,008^{* *}$ \\
& Huynh-Feldt & 205956,652 & 1,612 & 127764,358 & 8,778 & $0,006^{* *}$ \\
& Límite-inferior & 205956,652 & 1,000 & 205956,652 & 8,778 & $0,018^{*}$ \\
\hline \hline
\end{tabular}

\footnotetext{
* Significativo al 5\%; ** Significativo al $1 \%$.
} 
o con daño moderado, siendo ineficaz cuando hay un importante compromiso endotelial. De acuerdo a un trabajo de Gordo y colaboradores (1) la dorzolamida causa edema corneal irreversible en pacientes con glaucoma y antecedentes de prácticas quirúrgicas y compromiso endotelial previo.

Algunas comunicaciones (7) señalan que se comprometería la función endotelial de aquellas córneas con patología o con cirugías previas debido a que la farmacocinética se halla alterada en estos casos, llegando al endotelio concentraciones mayores a las habituales. La afectación corneal por acción de una droga puede evaluarse «in vitro» a través de la medición de las variaciones de potencial eléctrico transendotelial (8).

Nuestro trabajo pone de manifiesto la progresión del engrosamiento corneal en el transcurso de las mediciones para el ojo izquierdo tratado con dorzolamida $2 \%$ mientras que no hubo variaciones estadísticamente significativas en las corneas de conejos tratados con latanoprost $0,005 \%$.

Aunque la córnea del conejo puede no ser comparable a la humana; de acuerdo a un artículo de Kaufman H y col. el modelo de conejo de formación de humor acuoso no es confiable para los inhibidores de la anhidrasa carbónica y otras sustancias porque bloquearían la conocida capacidad de mitosis que tiene el endotelio corneal, ha servido para predecir la respuesta terapéutica ante la infección herpética con el uso de antivirales y corticoides, lo que avalaría el uso de estos animales en el presente estudio (9).

Este es un trabajo preliminar, tal vez no extrapolable de manera absoluta a la clínica de nuestros pacientes pero es una llamada de atención con respecto a la utilización de ciertas drogas que sabemos son seguras y efectivas en condiciones normales y pueden no serlo si se modifican ciertas condiciones como en una queratopatía severa.

La paquimetría ultrasónica es un método cuantitativo útil que nos permite determinar el espesor corneal tras queratoplastia penetrante. Debemos tener presente que la detección del incremento del mismo sobre los valores normales con la utilización de drogas antiglaucomatosas tiene un riesgo aumentado de fallo del injerto, siendo ésta una medida clínicamente relevante para observar la evolución de un transplante.

Tras 27 semanas de tratamiento, los ojos con queratoplastia, a los que se les suministró dorzolamida al $2 \%$ presentaron un engrosamiento corneal estadísticamente significativo en una proporción mayor respecto al grupo bajo latanoprost $0,005 \%$. La paquimetría ultrasónica nos permite la identificación y el seguimiento de estos cambios.

\section{BIBLIOGRAFÍA}

1. Domingo Gordo B, Urcelay Segura JL, Conejero Arroyo $J$, Balado Vázquez, P, Rodríguez Ausín P. Descompensación corneal en pacientes con compromiso endotelial tratados con dorzolamida tópica. Arch Soc Esp Oftalmol 2002; 77: 139-144.

2. Egan CA, Hodge DO, McLaren W, Bourne WM. Effect of dorzolamide on corneal endothelial function in normal human eyes. Invest Ophthalmol Vis Sci 1998; 39: 23-29.

3. Kaminski S, Hommer A, Koyuncu D, Biowski R, Barisani $T$, Baumgartner I. Influence of dorzolamide on corneal thickness, endothelial cell count and corneal sensibility. Acta Ophthalmol Scand 1998; 76: 78-79.

4. Lass JH, Khosrof SA, Laurence JK, Horwitz B, Ghosh K, Adamsons I. A double-masked, randomized, 1-year study comparing the corneal effects of dorzolamide, timolol and betaxolol. Dorzolamide Corneal Effects Study Group. Arch Ophthalmol 1998; 116: 1003-1010.

5. Giasson CJ, Tram Nguyen TQ, Boisjoly HM, Lesk M, Amyot M, Charest $M$. Dorzolamide and corneal recovery from edema in patients with glaucoma or ocular hypertension. Am J Ophthalmol 2000; 129: 144-150.

6. Borderie V, Touzeau O, Bourcier T, Allouch C, Zito E, Laroche L. Outcome of graft central thickness after penetrating keratoplasty. Ophthalmology 2005; 112: 626-633.

7. Konowal A, Morrison JC, Brown VL, Cooke DL, Maguire $J$, Verdier DV, et al. Irreversible corneal descompensation in patients treated with topical dorzolamide. Am J Ophthalmol 1999; 127: 403-406.

8. Akiyama R, Kuang K, Koniarek JP, Chiaradia P, Roberts $C W$, Fischbarg J. Solutions containing miotic agents: effects on corneal transendothelial electrical potential difference. Graefe's Arch Clin Exp Ophthalmol 1997; 235. 379-383.

9. Kaufman H, Varnell E, Toshida H, Kanai A, Thompson H, Bazan. Effects of topical Unoprostone and Latanoprost on acute an recurrent herpetic keratitis in the rabbit. $N \mathrm{Am} J$ Ophthalmol 2001; 131: 643-646. 